\title{
CdS Nanotubes and Y-Branched Nanochannels in AAM Fabricated by a Double Diffusion Route
}

\author{
Yong Lv, ${ }^{1}$ Weifeng Liu, ${ }^{1,2}$ Jianming Huang, ${ }^{1}$ and Lianzeng Yao ${ }^{1,2}$ \\ ${ }^{1}$ Department of Materials Science and Engineering, University of Science and Technology of China, Hefei, Anhui 230026, China \\ ${ }^{2}$ CAS Key Laboratory of Materials for Energy Conversion, Chinese Academy of Sciences, Hefei, Anhui 230026, China
}

Correspondence should be addressed to Weifeng Liu, liuwf@ustc.edu.cn

Received 31 March 2010; Revised 25 May 2010; Accepted 6 July 2010

Academic Editor: Bo Zou

Copyright ( 2011 Yong Lv et al. This is an open access article distributed under the Creative Commons Attribution License, which permits unrestricted use, distribution, and reproduction in any medium, provided the original work is properly cited.

\begin{abstract}
Polycrystalline CdS nanotubes and a novel partition nanostructure were prepared in an anodic alumina membrane (AAM) template using a double diffusion method at room temperature. Transmission electron microscopy (TEM), selected area electron diffraction (SAED) and energy dispersive X-ray spectroscopy (EDX) investigations indicate that the nanotubes consisting of CdS grains have been synthesized in the AAM nanoholes. The influence of the reagent concentration on the morphology of the nanotubes has been systemically studied using a field emission scanning electron microscope (FE-SEM). It is confirmed that the Y-branched nanochannels can only formed under certain concentrations of the reactants $\left(\mathrm{CdCl}_{2}\right.$ and $\mathrm{Na}_{2} \mathrm{~S}$ solutions). This novel Y-branched nanostructure may have potential applications for preparation of complicated nanostructure materials.
\end{abstract}

\section{Introduction}

One-dimensional (1D) semiconductor nanostructures have attracted tremendous attention because of their interesting chemical or physical properties and potential applications in nanodevices [1-3]. In the recent years, many studies have been focused on the CdS nanotubes and nanochannels owing to their large specific interfacial area, and various methods have been reported to synthesize the CdS nanotubes, such as the arc-electrodeposition technique [4], the CVD method [5, 6], the chemical bath deposition [7], the ultrasonic irradiation [8], the template methods [9-13], and so on. Among these methods, the double diffusion-template synthetic method is an effective and simple strategy to form CdS nanotubes.

Porous anodic alumina membranes (AAM) have been widely used as templates to prepare $1 \mathrm{D}$ nanostructural materials owing to their uniform and parallel porous structure. However, the morphology of the products crucially depends on the templates, that is, a normal AAM template can only be used to fabricate nanowires or nanotubes. As far as we know, few work focused on the complicated structures in the AAM. Lee et al. [14] reported a Y-branched nanochannel AAM template by adding a pore widening treatment between the second and the third anodization. Ho et al. [15] also fabricated multitiered branched porous anodic alumina (PAA) substrates consisting of an array of pores branching into smaller pores in succeeding tiers. These threedimensional nanostructures offer a new way to fabricate complicated nanomaterials in the AAM. In this paper, we report a facile way to obtain Y-branched nanochannels which may have potential applications for conformation of complicated nanomaterials.

\section{Experiments}

High-purity aluminum foil (purity $>99.999 \%$ ) annealed in a high-vacuum furnace was used in this work. A twostep anodic oxidation technique was applied to prepare the porous alumina template. The anodization was carried out in $0.3 \mathrm{M}$ oxalic acid with $40 \mathrm{~V}$ at a temperature around $0^{\circ} \mathrm{C}$. It took 10 and $24 \mathrm{~h}$ for the first and the second anodization, respectively. After the first-step anodization, the templates were immersed in a mixed solution containing $0.6 \mathrm{M} \mathrm{H}_{3} \mathrm{PO}_{4}$ and $0.15 \mathrm{M} \mathrm{H}_{2} \mathrm{CrO}_{4}$ at room temperature for $10 \mathrm{~h}$ to dissolve alumina. After the second anodization, a mixed solution of $1 \mathrm{M} \mathrm{CuCl}_{2}$ and $0.1 \mathrm{M} \mathrm{HCl}$ was used to remove the remaining aluminum metal. The barrier layer at the bottom 
of the membrane was dissolved to make a through-hole template, and the pores were widened in $0.5 \mathrm{M} \mathrm{H}_{3} \mathrm{PO}_{4}$ at room temperature. Then, the templates were immersed in deionized water to eliminate the residual $\mathrm{AlPO}_{4}$ and $\mathrm{H}_{3} \mathrm{PO}_{4}$. Finally, they were dried in an oven at $60^{\circ} \mathrm{C}$ for $4 \mathrm{~h}$.

The deposition experiments were carried out in homemade equipment having two equal troughs, and the schematic illustration is showed in Figure 1. In order to avoid air bubble formation in the channels, the membrane was immersed in deionized water and sonicated for a few minutes. Subsequently, the membrane was placed between the two troughs. A $\mathrm{Na}_{2} \mathrm{~S}$ solution $(0.01 \mathrm{M})$ was poured into one trough slowly. A few minutes later, a $\mathrm{CdCl}_{2}$ solution with a concentration varying from 0.003 to $0.01 \mathrm{M}$ was filled into the other one. Furthermore, the two liquid levels should be equal. The experiment was performed at room temperature for $1 \mathrm{~h}$. The templates turned yellow rapidly at the beginning. After completion of the reaction, the templates were thoroughly washed with deionized water and dried in the oven for characterization.

The morphology of the as-synthesized samples was observed using a field-emission scanning electron microscope (FE-SEM, JSM-6700F) and a transmission electron microscope (TEM, H-800). The energy dispersive X-ray spectroscope (EDX) attached on the TEM was employed to check the chemical composition of the as-prepared nanotubes. For TEM investigations, the samples were completely dissolved in a $2 \mathrm{M} \mathrm{NaOH}$ aqueous solution, washed with deionized water several times, and finally sonicated in ethanol for a few minutes.

\section{Results and Discussion}

Typical FE-SEM images of the AAM template are shown in Figure 2. It can be seen in Figure 2(a) that the AAM template possesses well-ordered cylindrical pores with a diameter of about $50 \mathrm{~nm}$. Figure 2(b) is the cross-section of the AAM template, which reveals that the channels are straight with smooth inner surface and the thickness of the walls is about $50 \mathrm{~nm}$.

Figure 3 demonstrates the morphology and structure of the as-prepared samples. The top-view of the surface immersed in the $\mathrm{CdCl}_{2}$ solution is shown in Figure 3(a). It is planar because the $\mathrm{CdCl}_{2}$ solution does not react with the AAM template. Comparing with the original AAM template (shown in Figure 2(a)), the diameter of the nanoholes is smaller and some of them are filled up, which implies that CdS has deposited in the pores, and the nanotubes are formed. The Y-branched nanostructures can be seen in Figure 3(b), and the boundary is smooth without any breaches. As the sample was slightly destroyed during preparation for FE-SEM observations, some defects could be found in the micrograph.

Figure 4 presents the TEM images and SAED patterns of the as-prepared CdS nanotubes. A single CdS nanotube is displayed in Figure 4(a). It can be observed that the nanotube is made of CdS grains, and single-crystal CdS nanotubes are hardly formed because of the inherent disadvantage of this method. The diameter of the CdS nanotube is about

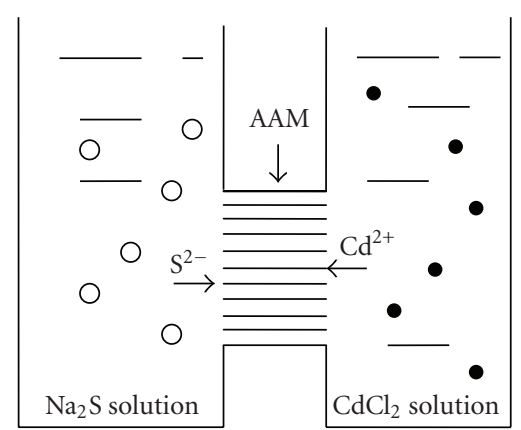

FIgURE 1: The schematic illustration of the double diffusion route experiment.

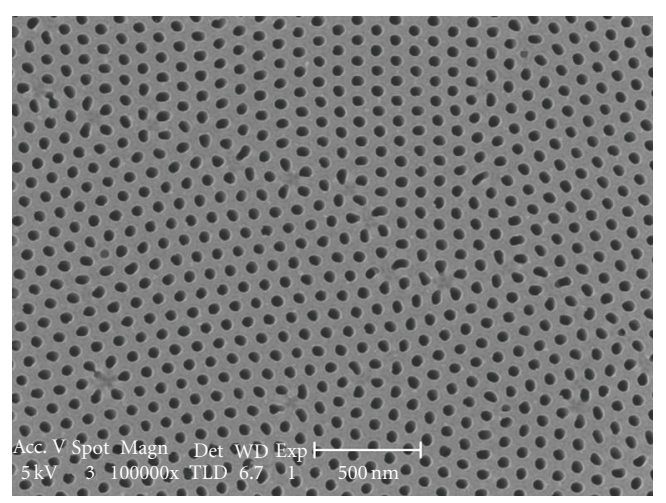

(a)

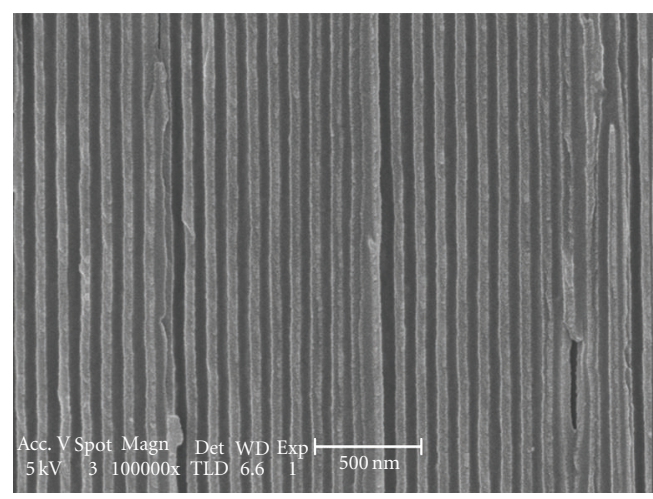

(b)

FIgURE 2: Typical FE-SEM images of the as-prepared AAM template. (a) top-view; (b) cross-section.

$50 \mathrm{~nm}$ consistent with the pore size of the AAM template. After sonication treatment, broken CdS nanotubes and CdS grains can be found in Figure 4(b). The EDX spectrum investigation of the as-synthesized samples (not showed here) reveals that only $\mathrm{S}, \mathrm{Cu}$, and $\mathrm{Cd}$ can be detected. The $\mathrm{Cu}$ signal in the spectrum originates from the copper grid, and the quantitative analysis indicates that the average atomic ratio of $\mathrm{Cd}: \mathrm{S}$ is near $1: 1$. Figure $4(\mathrm{c})$ show the SAED pattern of the nanotubes, which reveals that the samples have a polycrystalline structure with cubic spiauterite, and the 


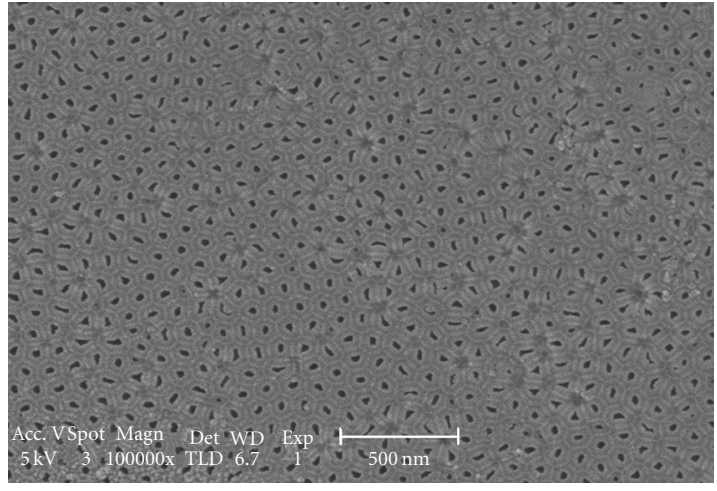

(a)

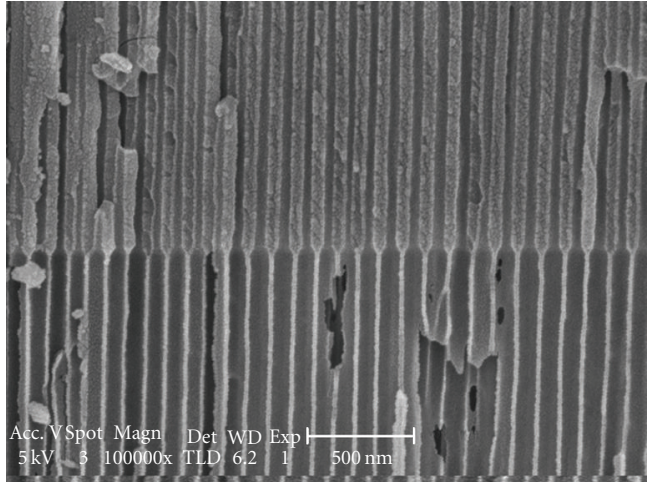

(b)

FIGURE 3: FE-SEM images of the as-synthesized sample under concentrations of $0.005 \mathrm{M} \mathrm{CdCl}_{2}$ and $0.01 \mathrm{M} \mathrm{Na} 2 \mathrm{~S}$. (a) top-view; (b) crosssection.

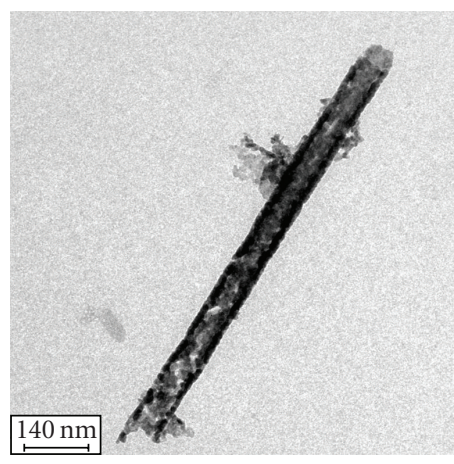

(a)

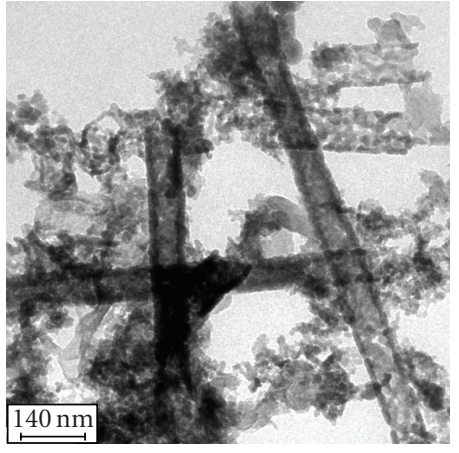

(b)

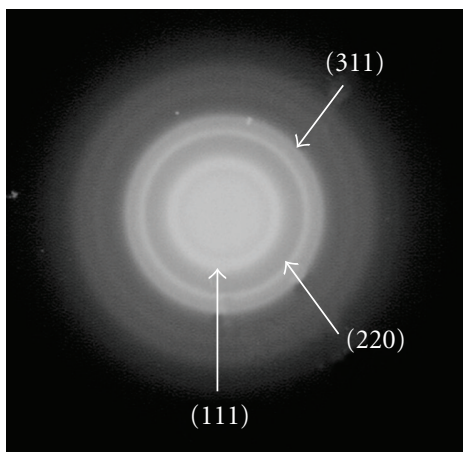

(c)

FIGURE 4: TEM images of the CdS nanotubes and their corresponding SAED pattern. (a) a single CdS nanotube; (b) some CdS nanotubes; (c) SAED pattern.

pattern can been indexed to (111), (220) and (311) lattice fringes.

In recent years, various nanomaterials were synthesized using this double diffusion method [16-19]. In fact, the essence of the double diffusion route is a chemical precipitation. Two different solutions are separated by the AAM template which can slow down the diffusion and the rate of crystallization to prevent overly rapidly mixing. The deposition occurs when $\mathrm{Cd}^{2+}$ and $\mathrm{S}^{2-}$ meet in the pores at the position where $\mathrm{C}_{\mathrm{Cd}^{2+}} * \mathrm{C}_{\mathrm{S}^{2-}}>\mathrm{Ksp}_{\mathrm{CdS}}$, where Ksp is the solubility of the product at the reaction temperature. The net reaction can be written as:

$$
\mathrm{Cd}^{2+}+\mathrm{S}^{2-} \longrightarrow \mathrm{CdS} \text {. }
$$

As the pore walls are positively charged, $\mathrm{S}^{2-}$ is likely to be adsorbed onto the walls [20].Then, the nucleation occurs on the walls and the crystallites finally form CdS nanotubes. If the reaction lasts long enough, the nanopores may be filled up and nanowires will be obtained [21].

Y-branched nanochannels would form when the two troughs filled with two different molar solutions $(0.01 \mathrm{M}$ $\mathrm{Na}_{2} \mathrm{~S}$ and $0.005 \mathrm{M} \mathrm{CdCl}_{2}$ ). In comparison with Figure 2(b), the pore diameter in Figure 3(b) upon the boundary is smaller, while that below it is much larger. The pore diameter below the boundary is nearly $100 \mathrm{~nm}$, almost two times of the original value. It is evident that the pores below the boundary are eroded by the $\mathrm{Na}_{2} \mathrm{~S}$ solution. As we know, $\mathrm{S}^{2-}$ has a larger ionic radius than $\mathrm{Cd}^{2+}$. Therefore, $\mathrm{Cd}^{2+}$ diffuses faster than $\mathrm{S}^{2-}$ in an aqueous solution, and $\mathrm{S}^{2-}$ is likely to aggregate in the pores near the $\mathrm{Na}_{2} \mathrm{~S}$ solution. And $\mathrm{S}^{2-}$, keeping diffusing into the AAM channels, not only to reacts with $\mathrm{Cd}^{2+}$ to form the CdS nanotubes but also makes the pores widened because the AAM is easy to be eroded in an alkalescence solution. Consequently, the Y-branched nanochannels form in the AAM.

The concentration of solutions is crucial to the fabrication of special nanostructures. In this work, the influence of the reactant concentrations on the morphology of the products was investigated by varying the $\mathrm{CdCl}_{2}$ concentration and keeping the other one unchanged $\left(0.01 \mathrm{M} \mathrm{Na}_{2} S\right)$. The $\mathrm{CdS}$ grains are deposited on the surface of the AAM template and no CdS nanotube is formed in the nanopores when the concentration of the $\mathrm{CdCl}_{2}$ solution decreases to $0.003 \mathrm{M}$. Since the $\mathrm{CdCl}_{2}$ concentration in the solution is so low, that very little $\mathrm{Cd}^{2+}$ can diffuse into the nanopores and react with $\mathrm{S}^{2-}$ to form CdS nanotubes. Therefore, $\mathrm{S}^{2-}$ travels straight 


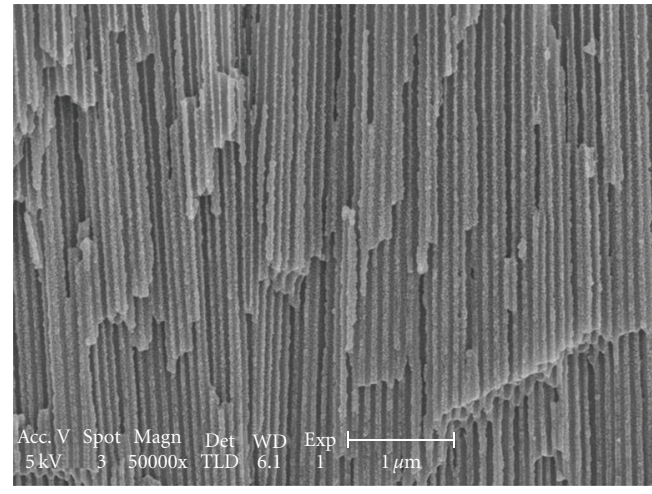

(a)

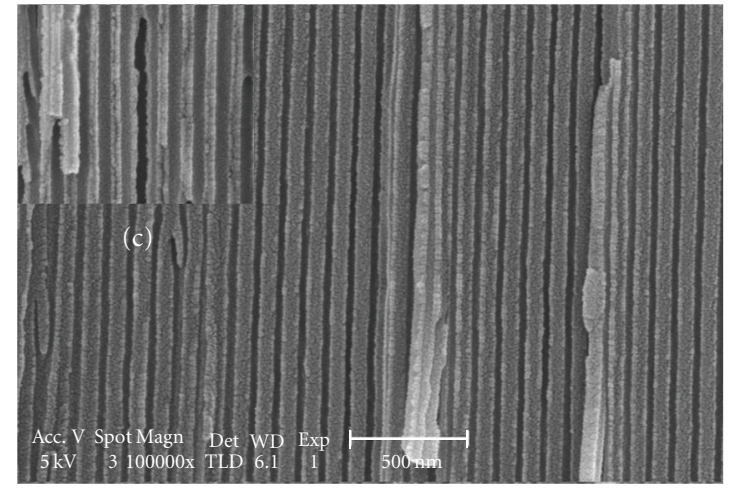

(b)

FIGURE 5: FE-SEM images of the prepared samples with different $\mathrm{CdCl}_{2}$ concentration. (a) $0.008 \mathrm{M}$; (b) $0.01 \mathrm{M}$; (c) magnification of (b).

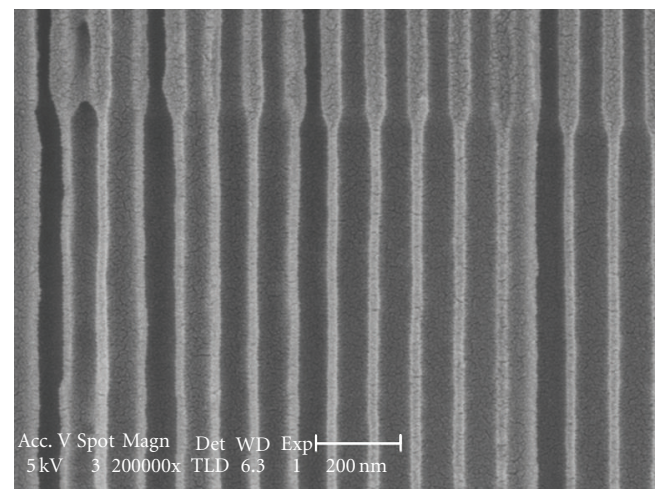

(a)

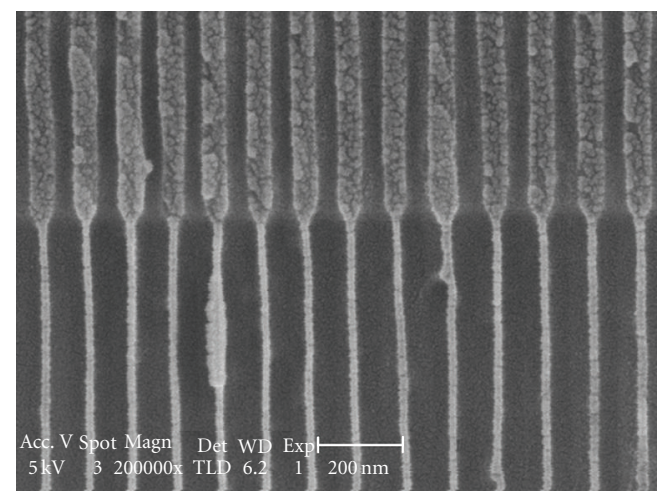

(c)

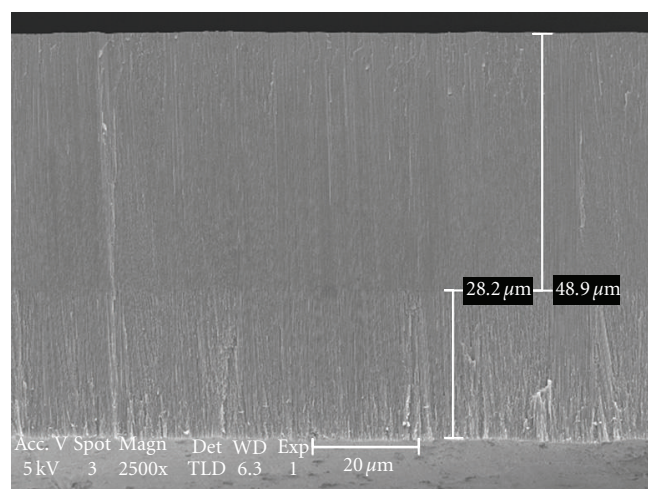

(b)

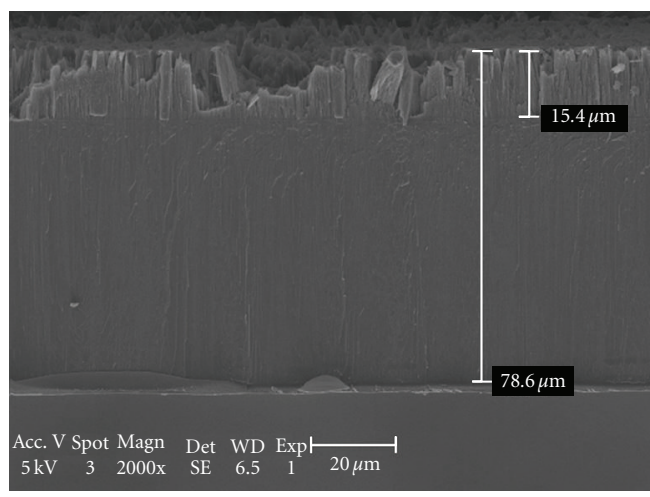

(d)

FIGURE 6: FE-SEM images of as-prepared samples under concentrations of $0.005 \mathrm{M} \mathrm{CdCl}_{2}$ and $0.01 \mathrm{M} \mathrm{Na}_{2} \mathrm{~S}$ with different reaction time (a, b) $40 \mathrm{~min}$; (c, d) $60 \mathrm{~min}$

through the nanopores and reacts with $\mathrm{Cd}^{2+}$ on the surface near the $\mathrm{CdCl}_{2}$ solution to produce CdS particles.

Figure 5 gives FE-SEM images of the samples prepared under different $\mathrm{CdCl}_{2}$ concentrations. It can be seen from Figure 5 that the nanotube diameter is obviously different at the two ends of the AAM template. The diameter of the nanotubes near the $\mathrm{Na}_{2} \mathrm{~S}$ solution is about $100 \mathrm{~nm}$, which is larger than that in the other end. It is found in Figure 5 that the diameter of the nanotubes in the end close to the $\mathrm{Na}_{2} \mathrm{~S}$ solution becomes small as the $\mathrm{CdCl}_{2}$ concentration increases. However, if the concentration of the $\mathrm{CdCl}_{2}$ solution is high enough $(>0.02 \mathrm{M})$, the CdS nanotubes cannot form 
in the pores. In the case of a high $\mathrm{CdCl}_{2}$ concentration, the diffusion rate of $\mathrm{Cd}^{2+}$ is so high that most of $\mathrm{Cd}^{2+}$ travels through the nanopores directly and reacts with $\mathrm{S}^{2-}$ to form the CdS particles on the surface of the AAM template immerging in the $\mathrm{Na}_{2} \mathrm{~S}$ solution.

When the reaction concentration of $\mathrm{CdCl}_{2}$ is $0.005 \mathrm{M}$ (the concentration of $\mathrm{Na}_{2} \mathrm{~S}$ was set at $0.01 \mathrm{M}$ ), Y-branched nanochannels with different diameter nanopores can be observed (see Figure 3(b)). The length and diameter of the Ybranched nanochannels can be varied with the reaction time. Figure 6 presents the FE-SEM photographs of the samples prepared with different reaction time. Figures 6(a) and 6(c) reveal that the pore diameter upon the boundary for the sample prepared for $60 \mathrm{~min}$ is smaller than that for $40 \mathrm{~min}$. The pore diameter of the sample below the boundary, on the contrary, is getting larger as the reaction time increases. The panorama of the two different samples is shown in Figures 6(b) and 6(d). It can be seen that, with increasing the reaction time, the corrosion proportion turns large and more $\mathrm{CdS}$ nanoparticles deposit on the walls of the nanochannels resulting in the small pore diameter. Moreover, the length of the CdS nanotubes in the AAM nanopores increases clearly with the reaction time, and the surface of the AAM membrane are destroyed as shown in Figure 6(d). This new nanostructure may have great potential applications for synthesis of complicated nanomaterials using an electrochemical deposition, a sol-gel template method and so on.

\section{Conclusion}

In summary, CdS nanotubes and Y-branched nanochannels templates have been prepared at room temperature using a double diffusion method. The as-prepared nanotubes consist of the CdS grains. It is found that the concentration of the reactants contributes to the formation of the special nanostructures. This Y-branched nanostructure may be used as a template to synthesize complicated nanomaterials in the AAM.

\section{References}

[1] A. M. Morales and C. M. Lieber, "A laser ablation method for the synthesis of crystalline semiconductor nanowires," Science, vol. 279, no. 5348, pp. 208-211, 1998.

[2] J. Hone, B. Batlogg, Z. Benes, A. T. Johnson, and J. E. Fischer, "Quantized phonon spectrum of single-wall carbon nanotubes," Science, vol. 289, no. 5485, pp. 1730-1733, 2000.

[3] M. S. Gudiksen, L. J. Lauhon, J. Wang, D. C. Smith, and C. M. Lieber, "Growth of nanowire superlattice structures for nanoscale photonics and electronics," Nature, vol. 415, no. 6872, pp. 617-620, 2002.

[4] C. Wang, J. Fang, J. He, and C. J. O'Connor, "Convenient arcelectrodeposition technique to synthesize CdS nanotubes at room temperature," Journal of Materials Science Letters, vol. 22, no. 6, pp. 413-415, 2003.

[5] X.-P. Shen, A.-H. Yuan, F. Wang, J.-M. Hong, and Z. Xu, "Fabrication of well-aligned CdS nanotubes by CVD-template method," Solid State Communications, vol. 133, no. 1,pp. 1922, 2005.

[6] H. Pan, C. K. Poh, Y. Zhu et al., "Novel CdS nanostructures: synthesis and field emission," Journal of Physical Chemistry C, vol. 112, no. 30, pp. 11227-11230, 2008.

[7] H. Zhang, X. Ma, J. Xu, and D. Yang, "Synthesis of CdS nanotubes by chemical bath deposition," Journal of Crystal Growth, vol. 263, no. 1-4, pp. 372-376, 2004.

[8] M. Shao, Z. Wu, F. Gao, Y. Ye, and X. Wei, "Surfactantfree route to hexagonal CdS nanotubes under ultrasonic irradiation in aqueous solution at room temperature," Journal of Crystal Growth, vol. 260, no. 1-2, pp. 63-66, 2004.

[9] T. Peng, H. Yang, K. Dai, X. Pu, and K. Hirao, "Fabrication and characterization of CdS nanotube arrays in porous anodic aluminum oxide templates," Chemical Physics Letters, vol. 379, no. 5-6, pp. 432-436, 2003.

[10] S.-M. Zhou, S. Feng, and L.-D. Zhang, "A two-step route to self-assembly of CdS nanotubes via electrodeposition and dissolution," European Journal of Inorganic Chemistry, no. 9, pp. 1794-1797, 2003.

[11] X. Li, H. Chu, and Y. Li, "Sacrificial template growth of CdS nanotubes from $\mathrm{Cd}(\mathrm{OH})_{2}$ nanowires," Journal of Solid State Chemistry, vol. 179, no. 1, pp. 96-102, 2006.

[12] D. Mo, J. Liu, H. J. Yao et al., "Preparation and characterization of CdS nanotubes and nanowires by electrochemical synthesis in ion-track templates," Journal of Crystal Growth, vol. 310, no. 3, pp. 612-616, 2008.

[13] Y. Piao, H. Lim, J. Y. Chang, W.-Y. Lee, and H. Kim, "Nanostructured materials prepared by use of ordered porous alumina membranes," Electrochimica Acta, vol. 50, no. 15, pp. 2997-3013, 2005.

[14] J. S. Lee, G. H. Gu, H. Kim, K. S. Jeong, J. Bae, and J. S. Suh, "Growth of carbon nanotubes on anodic aluminum oxide templates: fabrication of a tube-in-tube and linearly joined tube," Chemistry of Materials, vol. 13, no. 7, pp. 2387-2391, 2001.

[15] A. Y. Y. Ho, H. Gao, Y. C. Lam, and I. Rodríguez, "Controlled fabrication of multitiered three-dimensional nanostructures in porous alumina," Advanced Functional Materials, vol. 18, no. 14, pp. 2057-2063, 2008.

[16] Y. Mao and S. S. Wong, "General, room-temperature method for the synthesis of isolated as well as arrays of singlecrystalline ABO4-type nanorods," Journal of the American Chemical Society, vol. 126, no. 46, pp. 15245-15252, 2004.

[17] N. Wada, K. Yamashita, and T. Umegaki, "Effects of divalent cations upon nucleation, growth and transformation of calcium carbonate polymorphs under conditions of double diffusion," Journal of Crystal Growth, vol. 148, no. 3, pp. 297304, 1995.

[18] R. J. Park and F. C. Meldrum, "Shape-constraint as a route to calcite single crystals with complex morphologies," Journal of Materials Chemistry, vol. 14, no. 14, pp. 2291-2296, 2004.

[19] F. Peters and M. Epple, "Crystallisation of calcium phosphates under constant conditions with a double diffusion set-up," Journal of the Chemical Society, Dalton Transactions, no. 24, pp. 3585-3592, 2001. 
[20] O. Ikeda, M. Ohtani, T. Yamaguchi, and A. Komura, "Direct electrochemistry of cytochrome $\mathrm{c}$ at a glassy carbon electrode covered with a microporous alumina membrane," Electrochimica Acta, vol. 43, no. 8, pp. 833-839, 1997.

[21] S. P. Mondal, K. Das, A. Dhar, and S. K. Ray, "Characteristics of CdS nanowires grown in a porous alumina template using a two-cell method," Nanotechnology, vol. 18, no. 9, Article ID 095606, 2007. 

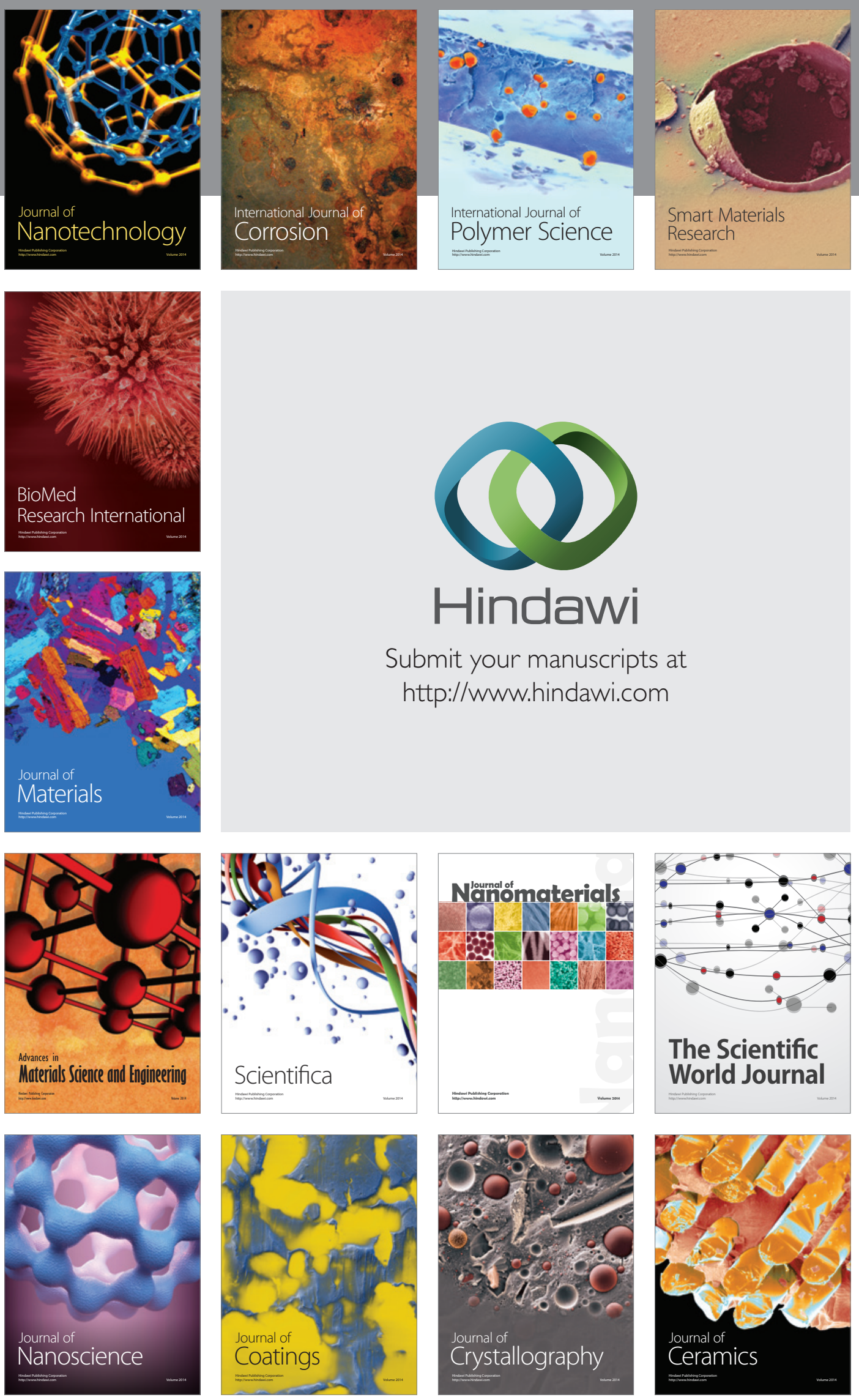

The Scientific World Journal

Submit your manuscripts at

http://www.hindawi.com

\section{World Journal}

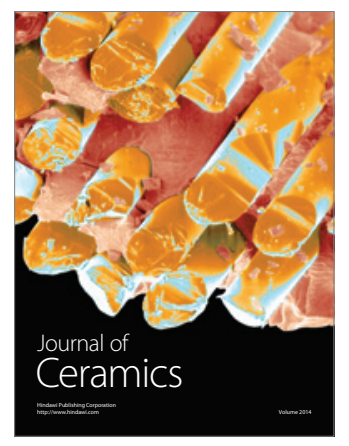

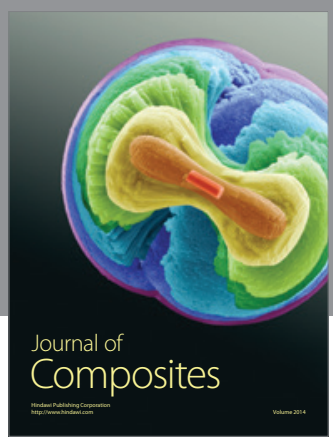
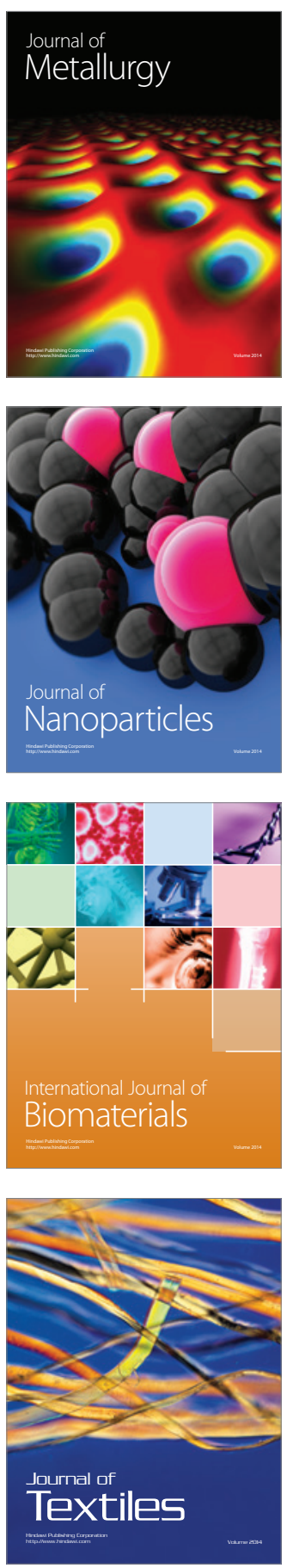